\title{
Duygu Analizi İçin Yeni Bir Sözlük; NAYALex Duygu Sözlüğü
}

\author{
Yakup Atl1 $^{1^{*}}$, Nagehan İlhan ${ }^{2}$ \\ 1*Harran Üniversitesi, Mühendislik Fakültesi, Bilgisayar Mühendisliği Bölümü, Şanlıurfa, Türkiye (ORCID: 0000-0002-8980-7243), yakupatli@ gmail.com \\ ${ }^{2}$ Harran Üniversitesi, Mühendislik Fakültesi, Bilgisayar Mühendisliği Bölümü, Şanlıurfa, Türkiye (ORCID: 0000-0002-1367-9230), nagehanilhan@ @harran.edu.tr
}

(İlk Geliş Tarihi 27 Temmuz 2021 ve Kabul Tarihi 10 Kasım 2021)

(DOI:10.31590/ejosat.974886)

ATIF/REFERENCE: Atlı, Y. \& İlhan, N. (2021). Duygu Analizi İçin Yeni Bir Sözlük; NAYALex Duygu Sözlüğü. Avrupa Bilim ve Teknoloji Dergisi, (27), 1050-1060.

\section{$\ddot{O} \mathbf{z}$}

İletişimin ayrılmaz parçası olan duygular, farklı şekillerde (konuşma, jestler, yüz ifadeleri vb.) ortaya çıkmaktadır. Sosyal paylaşım platformlarında ise insanlar duygu ve düşüncelerini en çok metinsel paylaşımlar ile ifade etmektedir. İnsanların sosyal medya aracıllı̆ 1 ile paylaştığı metinler kişilerin duygu durumları hakkında fikir vermektedir. Kişilik tespitinde duyguların sıklığının kişilik özellikleri ile ilişkili olduğunu gösteren birçok çalışma yapılmıştır. Dolayısıyla, sosyal medyada paylaşılan mesajlarda saklı olan duyguların tespiti ve ortaya çıkarılması önemlidir. Metinlerde saklı olan duygular kelime-duygu sözlükleriyle ortaya çıkarılabilmektedir. Bu sözlüklere baktığımızda en fazla sayıda duygu çıkarımı yapabilen NRC Duygu Sözlüğ̈̈, olumlu-olumsuz ile birlikte 8 farklı duyguyu ortaya çıkarabilmektedir. Ancak metin aracılı̆̆ 1 ile duygularını yansıtan kişilerin duygularını, olumlu-olumsuz veya birkaç farklı duygu ile sınırlı tutmak çoğu zaman kişilik tespitinde yetersiz kalmaktadır. Bu çalışmada, paylaşılan metinlerden daha fazla duygu yakalamak için metinden olumlu-olumsuz ile birlikte (umut, kaygı, sevgi, karamsarlık, iyimserlik, öfke, korku, üzüntü vb.) 38 farklı duygu çıkarımı yapan NAYALexsözlüğü önerilmektedir. NRC Duygu Sözlüğüne ve Plutchik'in Temel Duyguların Psikoevrimsel Teorisine dayandırdığımız sözlüğümüzün her bir kelimesi 38 farklı duygudan en az biri ile ilişkilendirilmiş 6469 İngilizce kelimeden oluşmaktadır. Instagram kullanıcı paylaşımlarına ait 10000 farklı paylaşımdan oluşan veri setimiz üzerinde birtakım deneyler yaparak, NAYALex sözlüğümüzün uygulanabilirliği ve kullanılabilirliği gösterilmiş̧ir. Diğer (LIWC, EmoSenticNet, NRC, Empath) duygu sözlükleriyle karşılaştırıldığında, sözlüğümüz Tiffany'nin belirttiği 154 duygu için \%24,7 ile en kapsamlı duyguyu tespit edebilir.

Anahtar Kelimeler: NRC, NAYALex, Duygu Analizi, Duygu Sözlüğü, Plutchik.

\section{A New Dictionary For Sentiment Analysis; NAYALex Emotion Dictionary}

\begin{abstract}
Emotions, which are an integral part of communication, emerge in different ways (speech, gestures, facial expressions, etc.). On social sharing platforms, people express their feelings and thoughts mostly with textual shares. Textual sharings of people through social media give an idea about their emotional state.Many studies have been carried out showing that the frequency of emotions in personality inference is related to personality traits.Therefore, it is important to detect and reveal the emotions hidden in the messages shared on social media.Emotions are hidden in textual posts that people share via social media. It is crucial to detect and reveal the emotions hidden in the messages shared on social media. When we look at these lexicons, the NRC Emotion Lexicon, which can detect the greatest number of emotions, can reveal 8 different emotions in total, positive and negative. However, limiting the emotions of people who reflect their feelings through text to positive-negative or a few different emotions is often insufficient in personality determination. In this study,the NAYALex lexicon that can detect 38 different emotions (hope, anxiety, love, pessimism, optimism, anger, fear, sadness, etc.) from texts is proposed to recognize more emotions from shared texts. NRC Emotion Lexicon and each word of our lexicon, which we base on Plutchik'sPsychoevolutionary Theory of emotions, consists of 6469 English words associated with at least one of 38 different emotions. The applicability and usability of our NAYALexLexiconis demonstrated by conducting some experiments on our data set consisting of 10000 different posts belonging to Instagram users.Compared to other (LIWC, EmoSenticNet, NRC, Empath) emotion lexicons, our lexicon can detect the highest comprehensive emotion with $24.7 \%$ for the 154 emotions Tiffany stated.
\end{abstract}

Keywords: NRC, NAYALex, Emotion Analysis, Emotion Lexicon, Plutchik.

*Sorumlu Yazar:yakupatli@gmail.com 


\section{Giriş}

İnternet kullanımının artmasıyla, insanlar arasındaki sanal iletişim ve etkileşim büyük artış göstermiştir. $\mathrm{Bu}$ artış beraberinde günümüzde popüler olan sosyal ağların ortaya çıkmasının temelini oluşturmaktadır. Dijital kullanımın artması ve buna paralel olarak sosyal medya kullanımının artması, sosyal medyayı araştırma noktası haline getirmiştir. Sosyal medya kullanıc1ları Facebook, Youtube, Instagram, Twitter, Google+ gibi birçok sosyal medya platformları aracılığı ile kişiliklerine ait duyguları barındıran paylaşımlar yapmaktadırlar. Bu durum sosyal medya platformlarını kullanan kişileri yakından takip eden işletme ve araştırma kuruluşlarının duyguların tespitine yönelmelerine sebep olmuştur. İşletme ve kuruluşlar sosyal medyada kullanıcılarının kişiliklerini dikkate alarak farklı strateji ve uygulamalar geliştirmektedirler. Sosyal medyada insanlar zevklerini, fikirlerini veya duygularını barındıran birçok paylaşım yapmaktadır. Sosyal medya, kullanıcıların duygularını yansıtan paylaşımlarıyla yararlı ve önemli bir büyük veri kaynağı haline gelmiştir (Hidalgo vd., 2015).

Yaşamımız üzerinde etkisi yadsınamayacak kadar önemli olan kişilik, geleneksel yöntem olan anketler ile belirlenmektedir. Kişiliğin, anketler yerine kişinin duygularını ve fikirlerini serbestçe ifade ettiği metinlerden ortaya çıkarılması daha çok tercih edilen bir durumdur. Duygu ve davranışlarla ortaya çıkan kişilik, biyolojik ve çevresel faktörlerden etkilenir. Kişiliğin duygularla ilişkili olduğunu ve bazı duygulara karşılık gelen sözcük gruplarının kişilikle ilişkili olduğu gösterilmiştir (Mohammad\&Kiritchenko, 2013).

Sosyal medya paylaşımlarındaki duyguları ortaya çıkarmak önemini korumakla birlikte, doğal dil işlemedeki belirsizlik ve karmaşıklık bu alandaki çalışmaları zorlaştırmaktadır (Hussein, 2018). Son zamanlarda kişilerin sosyal medyadaki paylaşımlarını dikkate alarak duyguları tespit etmeye yönelik birçok çalışma yapılmıştır. Literatürde sadece olumlu-olumsuz duygulara dayandırılan çalışmalar (örneğin, Alarid, 2016; Kušen vd., 2017; Woolf, 2016) daha fazla iken, bireysel duyguları (üzüntü, öfke, neşe) tanımlayan çok az çalışma yapılmıştır.

Duygular, kişilik ile ilişkilidir ve kişilik tespitinde önemli yere sahiptirler. Kişilik tespitine yönelik yapılan çalışmalarda, duygular olumlu- olumsuz olarak ele alınmakta ve sınırlı sayıda duygu ile yapılan çalışmalar analiz için yetersiz kalmaktadır. NRC (Mohammad, 2016), EmosenticNet (Poria vd., 2012), DepecheMood (Staiano\&Guerini, 2014), LIWC (Pennebaker vd., 2001) ve Empath (Fast vd., 2016) gibi sözlükler bu alanda kullanılan duygu sözlükleridir.

Duyguların sabit olmayan davranışlarla ortaya çıkması, insan kişiliği üzerinde etkisi olan duyguların belirlenmesini zorlaştırmıştır. Duyguların kişiliği belirlemedeki etkisi, duyguların doğru belirlenmesini zorunlu kılmaktadır. Sosyal medya kullanıcıları, duygularını paylaştıkları metinlere yansıtmakta ve metinler üzerinde yapılan çalışmalar için geniş veri havuzları sunmaktadır. Ortaya çıkan duyguların, metinselverilerden çıkarılması duygu sözlükleri ile yapilabilmektedir. NRC (Mohammad, NRC WordEmotionAssociationLexicon), EmosenticNet (Poria vd., 2012), DepecheMood (Staiano ve Guerini, 2014), LIWC (Pennebaker vd., 2001) ve Empath (Fast et al., 2016) gibi duygu sınıflandırması için kullanılan sözlüklerin dezavantajı, anahtar kelimelere atanan duygu ifadelerinin sinırlı sayıda duyguyu kapsamasıdır. Sınırlı sayıdaki duygu çıkarımı, kişiliğin belirlenmesi üzerinde olumsuz etkigöstermektedir.

Ayrık duygu teorisine göre, insanların doğuştan gelen bir dizi temel duyguya sahip olduğu düşünülmektedir (Colombetti, 2009). 1980'de Robert Plutchik, On Postulates'ten esinlenerek sekiz duygudan (neşe, güven, korku, şaşkınlık, üzüntü, tiksinti, öfke ve beklenti) oluşan bir duygu çarkı çizdi (Plutchik, 1980; Plutchik, 2001). Plutchik ayrıca iki duygudan oluşan ve "Birincil", "İkincil" ve "Üçüncül" ikililerden ve zıt duygulardan oluşan yirmisekiz farklı duyguyu ele alan temel duyguların psikoevrimsel teorisini ortaya attı (Drews\&Krohn, 2007). TiffanyWatt Smith dünya çapında 154 farklı duygu olduğunu belirtmiştir (Medhat vd., 2014).

Tiffany'nin belirttiği 154 farklı duygunun varlığı, sınırlı sayıda duygu çıkarımı yapan sözlüklerin duygu analizleri için sınırlı olabileceğini ve daha fazla duygu çıarımı yapabilen sözlüklere ihtiyaç duyulduğunu ortaya koymaktadır (Medhat vd., 2014).

Duygu analizi için kullanılan önceki sözlüklerden, NRC sekiz (Trust, Anticipation, Disgust, Joy, Fear, Surprise, Anger, Sadness), EmosenticNet alt1 (Disgust, Joy, Fear, Surprise, Anger, Sadness), LIWC dört (Postive, Negative, Sadness, Anger), Empath altı (Joy, Fear, Surprise, Anger, Sadness, Love) farklı duygu için sınıflandırma yapmaktadır.

Bu çalışmada, yaygın olarak kullanılan (NRC(Mohammad, 2016), EmosenticNet(Poria vd., 2012), DepecheMood(Staiano\&Guerini, 2014), LIWC(Pennebaker vd., 2001) ve Empath (Fast vd., 2016)) duygu sözlüklerinin ortaya çıkardığı duyguları kapsayan ve olumlu-olumsuz ile birlikte 38 farklı duygu sınıflandırması yapan NAYALex Duygu Sözlüğü önerilmektedir. NRC Duygu Sözlüğü ve Plutchik'in Temel Duyguların Psikoevrimsel Teorisine (Drews\&Krohn, 2007) dayandırdığımız NAYALex’teki her bir kelime 38 farklı duygu ile ilişkilendirilmiştir.

NAYALex sözlüğümüz ile Tiffany’nin belirttiği 154 farklı duygunun 38 farklı duygusu için çıkarım yapılabilmektedir. Bu çalışmada, kişiliğin belirlenmesinde büyük öneme sahip olan duygu sınıflandırmalarının literatürde 4 duygu(LIWC), 6 duygu(EmosenticNet, Empath) ve 8 duygu(NRC) gibi kisitl sayıda duygu çıkarımı yapan sözlüklerden kaynaklanan dezavantajın ortadan kaldırılması hedeflenmiş ve bunun için 38 farklı duygu için sınıflandırma yapabilen NAYALex sözlüğü oluşturulmuştur.

$\mathrm{Bu}$ çalışma sonucunda, sözlük tabanlı duygu sınıflandırması için doğrudan 38 farklı duyguyu ortaya çıkarabilen ilk en kapsamlı sözlük oluşturulmuş ve test edilmiştir.

Çalışmanın geri kalanı aşağıdaki gibi yapılandırılmıştır. Bölüm II'de ilgili çalışmalardan bahsedilmiştir. Bölüm III'te sözlüğün oluşturulması ayrıntılı olarak açıklanmıştır. Bölüm IV'te deneysel çalışmalar gösterilmiş ve sonuçları analiz edilmiştir. Bölüm V'de sonuçlar ve gelecekteki çalışmalar verilmiştir.

\section{LiteratürTaraması}

Duygu analizi, bireylerin metinler aracılığı ile yansıttığı duygularının belirli yordamlarla davranış, görüş, tutum ve duyguların istatistiksel yöntemlere göre gerçekleştirilen hesaplamalı analizlerdir (Medhat vd., 2014; Pang\& Lee, 2009). Bir kişinin üzerinde yorum yaptığı faktörler kişiler, konular, 
nesneler veya varlıklar olabilir. Duygu analizi ile herhangi bir kişi, konu, nesne veya varlık üzerinde görüş belirten kişinin duygusal durumunu tespit etmek amaçlanır (Cambria vd., 2013). Duygu analizi ile tutumların, değerlendirmelerin, görüşlerin, hislerin ve duyguların metinsel ifadelerden çıkarılması doğal dil işlemenin en önemli konularından biridir.

Etkileşimde etkili olan ve duyguların yansıdığı metinlerin yanında temel araçlardan biri de konuşmadır. Derin öğrenme modelleri kullanılarak tanıma sorunlarına çözümler üretilmektedir(Mittal vd., 2018; Bae vd., 2016; Malik vd., 2020).Konuşma duygularının ortaya çıkarılması ve sınıflandırılması için yapılan araştırmalar giderek ilgi görmeye başlamıştır. İnsanların akıllı sistemler ile etkileşimlerinin artması ve akıllı sistemlerin de veri işleme hızlarının ve performanslarının artmasıyladuyguların ortaya çıkarılmasında ilgiyi bu alana yöneltmiştir.

Konuşma duygularının ortaya çıkarılması için uygulanan geleneksel yöntemlerde konuşmanın akustik içeriği ile ilgili özellikler çıkarılmaktadır. Makine öğrenme teknikleri ve önceden belirlenmiş duygu etiketleri kullanılarak öznitelikleri çıkarılmış konuşma duyguları sınıflandırılabilmektedir. İnsanbilgisayar etkileşimi sonucunda konuşmadan duygu tanıma probleminde sınıflandırma doğruluğunu arttırmak için yeni bir hibrit mimari önermişlerdir(Er, 2020). Önerilen tekniğin konuşma duygularının doğru ve verimli bir şekilde sınıflandırılabilindiğini yapılan deneyler ile açık bir şekilde göstermişlerdir.

Öznel bir olgu olan duygular, boyutsal ve kategorik olmak üzere iki grupta ele alınmaktadır. Kategorik modellerde tek kelime ya da kelime grupları kullanılarak duygular tanımlanırken, iki boyutlu modellerde uyarılma değerleri ve değerlilik ölçüleri ele alınmaktadır(Alarcao\&Fonseca, 2017;
Lang, 1995). Farklı önerilerde bulunan psikologlar ve bilim insanları duyguları öfke, korku, iğrenme, üzülme, mutluluk ve neşe olarak 6 temel sınıf olacak şekilde ayırmışlardır(Picard,2000).

Müziğin dinleyicileri üzerinde etkisi olduğu ve duyguları ortaya çıkarmada etkili olduğu kabul görmektedir. Dinleme esnasında beyinde oluşan elektriksel sinyallerin duyguların ortaya çıkarılmasında daha gerçekçi sonuçlar ortaya koyduğu ile ilgili birçok çalışma yapılmıştır. Müzik parçalarının dinlenmesi ile ortaya çıkan duyguların sınıflandırılması ve tanınması problemini ele almışlardır(Er\&Çiğg, 2020). Bunun için Türk müziği parçalarını katılımcılara dinleterek ve bunun sonucunda katılımcıların beyinlerinde oluşan sinyalleri inceleyerek rahatlatıcı, gergin, hüzünlü ve mutlu duygularını tanımaya çalışmışlardır. Makine öğrenme algoritmaları kullanılarak sınıflandırmalar yapılmış ve elde edilen doğruluk oranları kullanılan yöntemlerinin performansınıolumlu yönde etkilediği gözlemlenmiştir.

Duygu sınıflandırması, duygu analizinin en önemli adımıdır ve son yıllarda en fazla ilgi gören çalışma alanlarından biri olmuştur (Lin vd., 2014).

Duygu sınıflandırması, belge (Yessenalina vd., 2010), cümle (Pang vd., 2002) ve kelime (Breck vd., 2007) düzeyinde gerçekleştirilebilir. Duygu sınıflandırması için genellikle farklı yöntemler kullanılmaktadır. Duygu analizinde sözlüğe dayalı, makine öğrenmesine dayalı ve hibrit olmak üzere farklı yöntemler ele alınmaktadır (Medhat vd., 2014). Duygu analizinde kullanılan algoritmalar ve sınıflandırma yöntemleri Şekil-1'deki gibi gösterilmiştir (Medhat vd., 2014; Ravi\&Ravi, 2015).

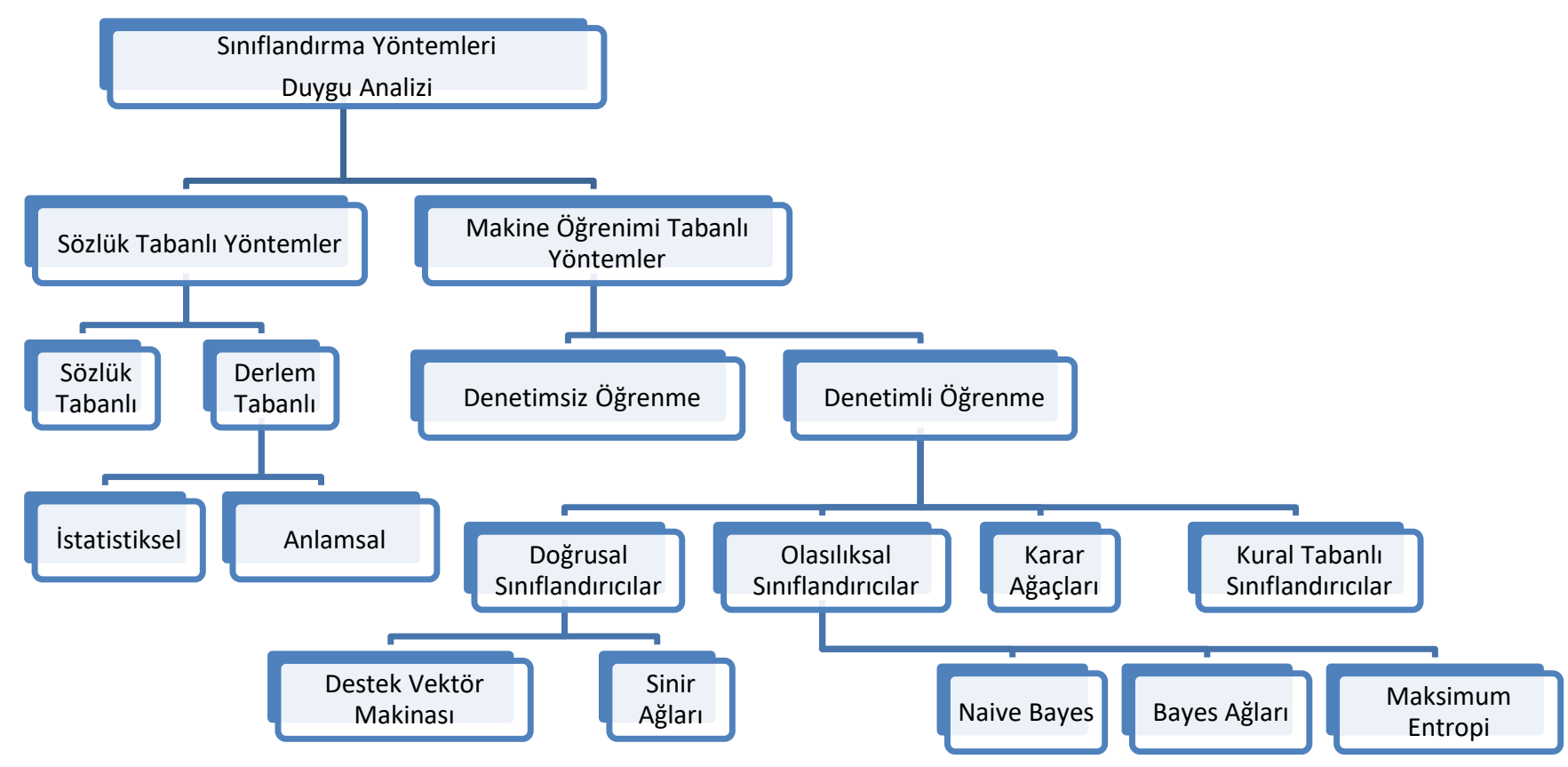

Şekil 1. Duygu analizinde kullanılan algoritmalar ve sınıflandırma yöntemleri (Medhat vd., 2014; Ravi\&Ravi, 2015). 
Makine öğrenmesi yaklaşımları dil yapılarının eğitilmesinde de kullanılmaktadır. Makine öğrenmesine dayalı yöntemlerin doğruluğu uygun eğitilme kalitesine, kullanılan sınıflandırıcının uygunluğuna ve kullanılan eğitim setine bağlı olarak değişebilmektedir (Devika vd., 2016). Makine öğrenmesine dayalı yöntemlerin eğitim seti vb. alanlara bağımlı olmaları ve eğitim gerektirmeleri gibi dezavantajları bulunmaktadır. Ancak sözlük temelli yaklaşımlar herhangi bir eğitim seti gerektirmez ve eğitim gerektirmemesi sözlük tabanlı yaklaşımlara avantaj sağlamaktadır.

Metin sınıflandırma için makine öğrenmesi yaklaşımları tercih edildiğinde denetimli ve denetimsiz öğrenme yöntemleri ele alınır. Denetimli öğrenmede, önceden etiketlenmiş bir veri kullanılarak bir sınıflandırma modeli oluşturulur. Daha sonra elde edilen sınıflandırma modeli, test verilerinin sınıf etiketi atamaları için kullanılır. Denetimli öğrenme yöntemi, makine öğrenmesi yaklaşımında daha fazla tercih edilmektedir. En yaygın olarak kullanılan denetimli öğrenme algoritmalarına baktığımızda, Olasılıksal Sınıflandırıcılar (NaiveBayes, Bayes Ağları, Maksimum-Entropi), Kural Tabanlı Sinıflandırıcılar, Doğrusal Sınıflandırıcılar(Destekçi Vektör Makinesi, Sinir Ağları) ve Karar Ağaçları yöntemleridir (Medhat vd., 2014) (Ravi\&Ravi, 2015).

Etiketli eğitim verilerine ulaşmanın zor olduğu durumlarda denetimsiz öğrenme yöntemleri uygulanır. Önceden belirlenmiş duygu terimlerinin bir araya getirilmesi sözlük tabanlı yaklaşımlara kaynak sağlar. Sözlük tabanlı yaklaşımların doğrulukları, oluşturulan sözlükteki kelime-duygu ikililerinin bulunma derecelerinden etkilenebilmektedir (Koumpouri vd., 2015). Kullanılan duygu sözlüğünün ölçeği, metinden çıkarılacak duyguları doğrudan etkilemektedir. Sözlük tabanlı yaklaşımda, önce metinden duygu kelimeleri çıkarılır ve çıkarılan duygu kelimelerinin eş ve zıt anlamları sözlükte aranır (Medhat vd., 2014). Derlem tabanlı yöntemler ise, anlamsal ve istatistiksel yaklaşımlar olmak üzere iki farklı şekilde ele alınır ve kelime kökleri dikkate alınarak duygu içeren kelimelerin metin içerisinden aranması şeklinde gerçekleştirilir (Medhat vd., 2014; Pang\& Lee, 2009). Hibrit yaklaşımlar, sözlük ve makine öğrenmesi yaklaşımlarının bir arada ele alınmasıyla oluşturulan yaklaşımlardır (Medhat vd., 2014; Liu, 2012). İstatistiksel yaklaşımda, istatistiksel yöntemler kullanılarak duygu ifade eden kelimelerin kökleri tespit edilir. Duygu, bir kelimenin pozitifnegatif cümle veya metin içinde bulunma sıklığı ile belirlenir. Kelimenin negatif veya pozitif cümlelerde bulunma frekans1, kelimenin pozitif, negatif veya nötr duygu bildiren bir kelime olması üzerinde etkilidir. Bir duyguyu ifade eden bir sözcükle bir kelimenin bir arada bulunma sıklığının daha yüksek olması, kelimenin benzer bir duyguya sahip olabileceği anlamına gelir(Medhat vd., 2014; Liu, 2012). Anlamsal yaklaşım, sözlük modeli oluşturma amacıyla benzer duygu içeren kelimelerin anlamsal olarak benzer olduğu temeline dayanır (Liu, 2012).

Sözlük tabanlı yaklaşımda, duyguların çıkarılması için sözcük kaynakları kullanılır. Önceden elde edilmiş bir kelime havuzuna göre metinden duygu ifade eden kelimeler ve duyguların eşleştirilmesi şeklinde gerçekleştirilir. Sözlük tabanlı yaklaşımlarda, hem MPQA (Deng\&Wiebe, 2015) ve SentiWordNet (Baccianella vd., 2010) gibi duygu sözlüklerinden hem de dil özelliklerinden faydalanırlar.

Sözlük tabanlı yaklaşımları performans olarak değerlendirdiğimizde, sözlükteki duygu ifadelerinin kutupluluk derecesine bağlı olarak değiştiği görülmektedir (Turney, 2002).
Sözlük tabanlı yaklaşımlarda, manuel olarak oluşturulan sözlüklerin yüksek maliyetinden kaçınmak için sözlüklerin otomatik bir şekilde oluşturulması önem kazanmıştır (Li vd., 2012; Hatzivassiloglou\&McKeown, 1997). Duygu sözlüklerinin genişletilmesi için kelimelerin dilsel ilişskileri veya anlamsal sözlükleri (WordNet) kullanan birçok çalışma olmuştur (Rao\&Ravichandran, 2009; Kamps vd., 2004). NRC duygu sözlüğü, kitle yaklaşımı ile oluşturulan bir sözlüktür (Mohammad vd., 2015). DepecheMood, kapsam açısından geniş ve yüksek hassasiyetli otomatik olarak oluşturulmuş bir sözlüktür (Staiano\&Guerini, 2014). LIWC sözlüğü olarak bilinen Dilbilimsel Sorgulama ve Kelime Sayımı, geniş bir metin analiz yazılımıdır (Pennebaker vd., 2001). EmoSenticNet, SenticNet kavramlarına altı WordNetAffect duygu etiketi atayan sözcüksel bir kaynaktır (Poria vd., 2012). Empath, büyük duygusal kategori kümelerini analiz etmek için tasarlanmış kelime sınıflandırma yaklaşımıdır. Bununla birlikte, F. Koto ve M. Adrianitwitter verilerini kullanarak ve Plutchik'in duygu çarkına (Plutchik, 2001) atıfta bulunarak Hashtag Tabanlı Duygu (HBE) sözlüğünü oluşturmuşlardır (Koto\&Adriani, 2015). Song ve arkadaşları 2016 yılında duygu-kelime sözlüğü kavramını, duyguların anlambilimini işin içine katarak genişletmişlerdir.

$\mathrm{Bu}$ çalışmamızda önerdiğimiz NAYALex sözlüğü, NRC Emotion sözlüğü ve Plutchnik'in duygu çarkıdâhil edilerek diğer sözlüklere göre daha fazla duygu sınıflandırması için oluşturulmuş ve kullanıma hazır hale getirilmiştir.

\section{Materyal ve Metod}

Sosyal medyanın yaygın bir şekilde kullanımıyla paylaşılan içeriklerdeki duyguların ortaya çıkarılması giderek önem kazanmaktadır. Sosyal medya kullanıcıları duygularını, paylaştıkları metinlere yansıtarak bu alanda yapılacak çalışmalar için büyük veri kaynakları oluşturmaktadırlar. Farklı şekillerde ortaya çıkan duyguların, metinlerden çıkarılması duygu sözlükleri ile yapılabilmektedir. Ancak duygu sınıflandırması için kullanılan sözlüklere baktığımızda, anahtar kelimelere atanmış duygu ifadelerinin sınırlı sayıdaki duyguları içermesi dezavantaj olarak görülmektedir.

\subsection{Veri Kümesi}

Deneysel işlemlerde kullanılan veri seti, hesabı gizli olmayan Instagram kullanıcilarına ait toplam 10000 veridenoluşmaktadır. Her bir veri herhangi bir kullanıcıya ait paylaşım bilgisini içermektedir. Instagram API kullanılarak elde edilen Instagram kullanıcılarına ait veriler toplanırken sadece İngilizce dilinde ve en az 5 kelimeden oluşan metinsel paylaşımlar dikkate alınmıştır. Elde edilen veri seti üzerinde doğal dil işleme teknikleri kullanılarak ön işlemler ve veri temizleme işlemleri yapılmıştır.

\subsection{Yöntem}

$\mathrm{Bu}$ çalışmada, kişiliğin belirlenmesinde büyük önemi olan duygu sınıflandırmalarının literatürde kısıtlı sayıda yapılabilmesinden kaynaklanandezavantajın ortadan kaldırılması hedeflenmiştir. 38 farklı duygu sınıflandırması yapabilen NAYALex sözlüğünü, Şekil-2'de gösterildiği gibi 3 ana aşamadaÖn İşlem Aşaması, Dönüşüm Aşaması ve SınıflandırmaSözlük Oluşturma Aşaması'yla oluşturduk. 


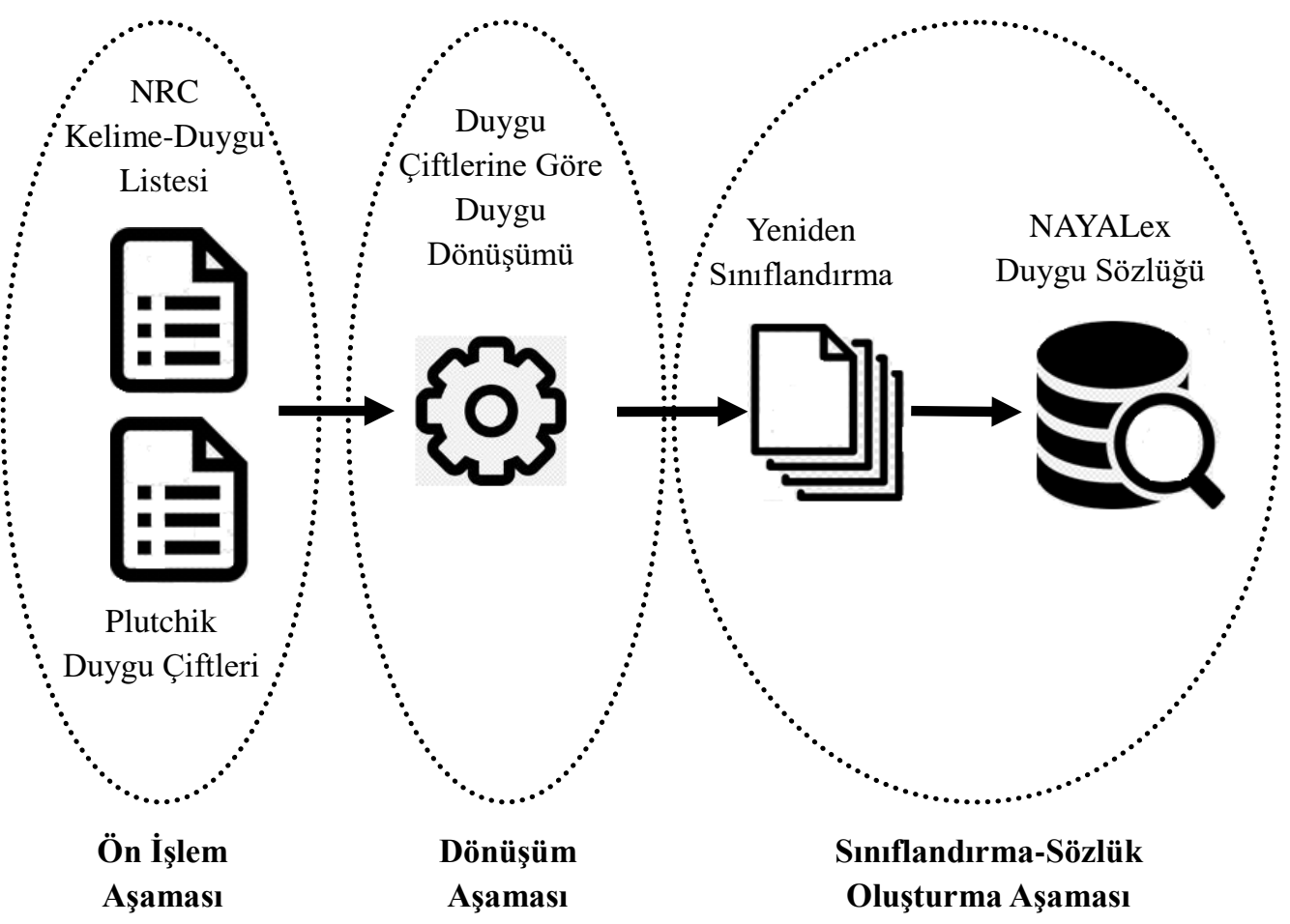

Şekil 2.NAYALex Duygu Sözlüğü Oluşturma Mimarisi

Ön İșlem Așaması: NRC Duygu Sözlüğündeki 14.182 kelime, duygu dönüşümü için bir ilişki derecesi atayarak NRC duygu kategorileri ile eşleştirilmiştir (Tablo-1). Aynı zamanda, NRC duygu kategorileri, Plutchik'in duygu kategorilerini oluşturmak için birleştirilir (Tablo-2). Bir sonraki dönüşüm aşaması için NRC ikili Duygu kombinasyonları ve bunun sonucunda Plutchik'in duygu kategorileri oluşturulur.

Tablo 1. NRC Duygu Sözlüğü (Mohammad, 2011)

\begin{tabular}{|c|c|c|c|}
\hline Sözlük & Kelime Sayısı & $\begin{array}{c}\text { Duygu } \\
\text { Kategorileri }\end{array}$ & $\begin{array}{l}\text { İlişkilendirme } \\
\text { Dereceleri }\end{array}$ \\
\hline & $\begin{array}{l}\text { 14.182 unigram } \\
\text { (kelime) }\end{array}$ & $\begin{array}{l}\text { duygular: } \\
\text { * olumlu } \\
\text { *olumsuz }\end{array}$ & $\begin{array}{l}0 \text { (ilişkili değil) } \\
\text { veya } 1 \text { (ilişkili) }\end{array}$ \\
\hline $\begin{array}{l}\text { NRC Sözcük-Duygu } \\
\text { Derneği Sözlüğü (NRC } \\
\text { Duygu sözlüğü veya } \\
\text { EmoLex olarak da } \\
\text { adlandırılır) }\end{array}$ & 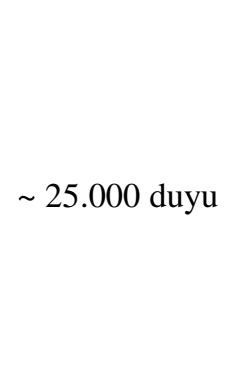 & $\begin{array}{l}\text { duygular: } \\
\text { *öfke } \\
\text { *beklenti } \\
\text { *tiksinti } \\
\text { *korku } \\
\text { *sevinç } \\
\text { *üzüntü } \\
\text { *sürpriz } \\
\text { *güven }\end{array}$ & $\begin{array}{l}\text { İlişkili değil, zayıf, } \\
\text { orta veya güçlü bir } \\
\text { şekilde ilişkili }\end{array}$ \\
\hline
\end{tabular}


Tablo 2.Plutchik'in Duygu Çiftleri (Drews, 2007)

\begin{tabular}{|c|l|l|c|l|l|}
\hline$\#$ & $\begin{array}{c}\text { İkili Duygu } \\
\text { Kombinasyonları }\end{array}$ & \multicolumn{1}{|c|}{ Duygular } & $\#$ & $\begin{array}{c}\text { İkili Duygu } \\
\text { Kombinasyonları }\end{array}$ & \multicolumn{1}{|c|}{ Duygular } \\
\hline 1 & Beklenti + Sevinç & İyimserlik, Cesaret & 15 & Sürpriz + Üzüntü & $\begin{array}{l}\text { Onaylanmama, Hayal } \\
\text { Kırıklı̆̆ }\end{array}$ \\
\hline 2 & Beklenti + Güven & Umut, Kadercilik & 16 & Sürpriz + Tiksinme & İnançsızlık, Şok \\
\hline 3 & Beklenti + Korku & Kayg,, Dehşet & 17 & Sürpriz + Öfke & Öfke, Nefret \\
\hline 4 & Sevinç + Güven & Sevgi, Dostluk & 18 & Üzüntü + Tiksinme & Pişmanlık, Sefalet \\
\hline 5 & Sevinç + Korku & Suçluluk, Heyecan & 19 & Üzüntü + Öfke & Kıskançlık, sıkıcılık \\
\hline 6 & Sevinç + Sürpriz & Keyif, Kader & 20 & Üzüntü + Beklenti & Karamsarlık \\
\hline 7 & Güven + Korku & Teslimiyet, Tevazu & 21 & Tiksinme + Öfke & Küçümseme \\
\hline 8 & Güven + Sürpriz & Merak & 22 & Tiksinme + Beklenti & Alaycılık \\
\hline 9 & Güven + Üzüntü & Duygusallık, Tevekkül & 23 & Tiksinme + Neşe & Hastalık, Kötülük \\
\hline 10 & Korku + Sürpriz & Huşu, Telaş & 24 & Öfke + Beklenti & Saldırganlık, İntikam \\
\hline 11 & Korku + Üzüntü & Umutsuzluk & 25 & Öfke + Sevinç & Gurur, Zafer \\
\hline 12 & Korku + Tiksinme & Utanç, Küstahlık & 26 & Öfke + Güven & Hakimiyet \\
\hline 13 & Sevinç + Üzüntü & Acı tatlllık & 27 & Korku + Öfke & Donukluk \\
\hline 14 & Güven + Tiksinme & Kararsılık & 28 & Sürpriz + Beklenti & Bilinç bulanıklı̆̆, şaşkınlık \\
\hline
\end{tabular}

Dönüșüm Așaması: NRC Duygu sözlüğündeki her bir kelime, olumlu-olumsuz duygularve 8 farklı duygu (neşe, güven, korku, şaşkınlık, üzüntü, tiksinme, öfke ve beklenti) için 0 (ilişki yok) ve 1 (ilişki var) şeklinde derecelendirilmiştir (Mohammad, 2016). Ön işlem aşamasında elde edilen duygu setleri referans alınarak, Plutchik duygu çiftlerinin etki ettiği ve ortaya çıkardığı yeni duygularla eşleştirilmektedir.

Sınıflandırma-Sözlük Olușturma Așaması: NRC Duygu Sözlüğü'nde bulunan 8 duygu ile Plutchik'in Temel Duyguların Psikoevrimsel Teorisi'ne (Drews\&Krohn, 2007) göre belirlenen 8 temel duygu aynıdır. Plutchik Duygu Çarkı'nda bulunan 8 temel duygu birbirleriyle birleşerek 28 duygu çifti oluşturmakta ve bu duygu çiftlerinin birleşimi daha farklı duyguları meydana getirmektedir. Tablo 2'deki İkili Duygu Kombinasyonları dikkate alınarak, duygu çiftleri için eşleştirmeler yapılmaktadır. NRC Duygu Sözlüğü'nde bulunan her bir kelimenin ilişkili olduğu duygular, Plutchik'in duygu çiftlerine göre uyarlanmakta ve 28 farklı duygudaki her bir kelime Plutchik'inTablo 2'deki duygu kategorileriyleyeniden ilişkilendirilmektedir. $\mathrm{Bu}$ ilişkilendirme şu şekilde yapılmaktadır;28 farklı duygu çifti için NRC Duygu Sözlüğü'ndeki her kelime aranmakta ve bulunan her duygu çiftine karşılık gelen duygu için 0 ve 1 şeklinde derecelendirilmektedir. Elde edilen kelime-duygu sözlüğündeki her kelime, ayrıca NRC Duygu sözlüğünde doğrudan karşılık bulan olumlu-olumsuz ve 8 farklı duygu için derecelendirilerek 38 farklı duygu içeren sözlük ortaya çıkarılmaktadır.

NAYALex sözlüğünün oluşturulması Tablo-3'te sembolik olarak ifade edilmiştir. Sembolik gösterimdeki (X,Y,Z) birer kelime, (A,B,C) NRC sözlüğünde bulunan ve(X,Y,Z) kelimeleriyle ilişsili olan duygular, $(\mathrm{A}+\mathrm{B}, \mathrm{B}+\mathrm{C}, \mathrm{A}+\mathrm{C})$ Plutchik teorisinde yer alan duygu çiftleri, $(\mathrm{J}, \mathrm{K}, \mathrm{L})$ duygu dönüşümü sonucu ortaya çıkan ve NAYALex sözlüğünde bulunan duyguları belirtmektedir.

NRC Duygu sözlüğünde bulunan her bir kelime (örneğin "X" kelimesi); NRC Duygu Sözlüğünde ilişkili olduğu duygular (A,B) için " 1 ", ilişkili olmadığ 1 duygular (C) için " 0 " ile belirtilmiştir. " $\mathrm{X}$ " kelimesi için duygu dönüşümüne etki eden Plutchik duygu çifti/çiftleri A $+\mathrm{B} \rightarrow \mathrm{J}$ şeklinde ifade edilmiştir. Duygu dönüşümü sonrası oluşan NAYALex sözlüğümüzde " $X$ " kelimesi için oluşan duygu ilişkileri $\mathrm{A} \rightarrow 1, \mathrm{~B} \rightarrow 1, \mathrm{C} \rightarrow 1, \mathrm{~J} \rightarrow$ $1, \mathrm{~K} \rightarrow 0, \mathrm{~L} \rightarrow 0$ şeklinde olmaktadır. 
Tablo 3. Duygu Çiftlerine Göre Duygu Dönüşümü ve Yeniden Sınıflandırma Sembolik Gösterimi

\begin{tabular}{|c|c|c|c|}
\hline $\begin{array}{l}\text { Sözlük Kelimesi } \\
\text { (X,Y,Z birer kelime) }\end{array}$ & $\begin{array}{l}\text { NRC Duygu Etiketi } \\
\text { (A,B,C birer duygu) }\end{array}$ & $\begin{array}{c}\text { Dönüşüme Etki Eden } \\
\text { Plutchik Duygu Çifti } \\
\text { (J,K,L oluşturulan yeni } \\
\text { duygular) }\end{array}$ & $\begin{array}{c}\text { NAYALex'te Oluşan Duygu } \\
\text { Etiketleri } \\
\begin{array}{c}\text { ilişkili } \\
1 \rightarrow \text { ilgili duygu sözlük kelimesi ile } \\
\text { ilisk } \\
0 \rightarrow \text { ilgili duygu sözlük kelimesi ile } \\
\text { ilişkili değil }\end{array} \\
\end{array}$ \\
\hline$X$ & $\begin{array}{l}\mathrm{A} \rightarrow 1 \\
\mathrm{~B} \rightarrow 1 \\
\mathrm{C} \rightarrow 0\end{array}$ & $\mathrm{~A}+\mathrm{B} \rightarrow \mathrm{J}$ & $\begin{array}{l}\mathrm{A} \rightarrow 1 \\
\mathrm{~B} \rightarrow 1 \\
\mathrm{C} \rightarrow 0 \\
\mathrm{~J} \rightarrow 1 \\
\mathrm{~K} \rightarrow 0 \\
\mathrm{~L} \rightarrow 0\end{array}$ \\
\hline $\mathrm{Y}$ & $\begin{array}{l}\mathrm{A} \rightarrow 0 \\
\mathrm{~B} \rightarrow 1 \\
\mathrm{C} \rightarrow 1\end{array}$ & $\mathrm{~B}+\mathrm{C} \rightarrow \mathrm{L}$ & $\begin{array}{l}\mathrm{A} \rightarrow 0 \\
\mathrm{~B} \rightarrow 1 \\
\mathrm{C} \rightarrow 1 \\
\mathrm{~J} \rightarrow 0 \\
\mathrm{~K} \rightarrow 0 \\
\mathrm{~L} \rightarrow 1\end{array}$ \\
\hline $\mathrm{Z}$ & $\begin{array}{l}\mathrm{A} \rightarrow 1 \\
\mathrm{~B} \rightarrow 1 \\
\mathrm{C} \rightarrow 1\end{array}$ & $\begin{array}{l}\mathrm{A}+\mathrm{B} \rightarrow \mathrm{J} \\
\mathrm{A}+\mathrm{C} \rightarrow \mathrm{K} \\
\mathrm{B}+\mathrm{C} \rightarrow \mathrm{L}\end{array}$ & $\begin{array}{l}\mathrm{A} \rightarrow 1 \\
\mathrm{~B} \rightarrow 1 \\
\mathrm{C} \rightarrow 1 \\
\mathrm{~J} \rightarrow 1 \\
\mathrm{~K} \rightarrow 1 \\
\mathrm{~L} \rightarrow 1\end{array}$ \\
\hline
\end{tabular}

Tablo 4. NRC ve NAYALex Kelime-Duygu Eşleştirmesi

\begin{tabular}{|c|c|c|}
\hline $\begin{array}{c}\text { Sözlükte Bulunan } \\
\text { Kelime }\end{array}$ & \multicolumn{2}{|c|}{ İlişkili Olduğu Duygular } \\
\cline { 2 - 3 } & NRC Sözlüğü & NAYALex Sözlüğü \\
\hline tribulation (sıkıntı) & Negatif, Korku, Üzüntü & Negatif, Korku, Üzüntü, Umutsuzluk \\
\hline palsy (acizlik) & $\begin{array}{c}\text { Negatif, Tiksinme, } \\
\text { Korku, Üzüntü }\end{array}$ & $\begin{array}{c}\text { Negatif, Tiksinme, Korku, Üzüntü, Utanç, } \\
\text { Pişmanlık, Umutsuzluk }\end{array}$ \\
\hline accolade (övgü) & $\begin{array}{c}\text { Pozitif, Beklenti, } \\
\text { Sevinç, Sürpriz, Güven }\end{array}$ & $\begin{array}{c}\text { Pozitif, Beklenti, Sevinç, Sürpriz, Güven, } \\
\text { Iyimserlik, Şaşınlık, Umut, Keyif, Sevgi, Merak }\end{array}$ \\
\hline mighty (güçlü) & $\begin{array}{c}\text { Pozitif, Öfke, Korku, } \\
\text { Sevinç, Güven }\end{array}$ & $\begin{array}{c}\text { Pozitif, Öfke, Korku, Sevinç, Güven, Donukluk, } \\
\text { Gurur, Hâkimiyet, Heyecan, Tevazu, Sevgi }\end{array}$ \\
\hline
\end{tabular}

NRC ve NAYALex sözlükleri için kelimelerin geçtiği metinleri ilişkili (1) etki gösterdiği duygular Tablo-4'te örnek olarak gösterilmiş̧ir. Yukarıda sembolik olarak oluşumu ifade ettiğimiz NAYALex sözlüğümüz için Python dilinde bir kütüphane oluşturduk. Oluşturduğumuz kütüphaneyi kullanarak sözlüğümüzün uygulanabilirliğini göstermek için Bölüm IV'te birtakım deneysel çalışmalar yaptık.

\section{Deneysel Çalışmalar ve Tartışma}

Bu bölümde NRC duygu Sözlüğ̈̈ (Mohammad, 2016) ve Plutchik'in Temel Duyguların Psikoevrimsel Teorisine (Drews\&Krohn, 2007) dayanarak melez bir şekilde oluşturulan
NAYALex sözlüğünün veri seti üzerinde uygulanması ve değerlendirilmesi hedeflenmektedir.

NAYALex sözlüğü için oluşturulanPython Kütüphanesi kullanılarak Instagram veri setindeki tüm veriler 38 farklı duygu için sınıflandırılmıştır. Sınıflandırılmış veri setinden istenilen bir kullanıcıya ait duygu yoğunluğunu ortaya çıkarmak için, veri setinde ilgili kullanıcıya ait sınıflandırılmış tüm verilerden ortalama duygu yoğunluğu elde edilmektedir. Şekil 3 'te 13 faklı kullanıcıya ait Instagram verilerinin kullanıcı bazlı olarak ortalama duygu yoğunluklarını gösterilmektedir. 
Şekil 3'te verilen ortalama duygu yoğunluğu 10000 farklı veriden oluşan veri setinde yapılan duygu sınıflandırması sonucu rasgele seçilmiş 13 kullanıcıya aittir.

Burada ifade edilen ortalama duygu yoğunluğu için, her bir kullanıcıya ait farklı sayıdaki paylaşımlarNAYALex Sözlüğü ile sınıflandırılmıştır. Ardından veri setindeki tüm paylaşımlar her bir kullanıcı için filtrelenerek ortalama duygu yoğunlukları elde edilmiştir. İstenilen kullanıcıların duygu yoğunlukları Şekil3 'teki gibi aynı anda gösterilerek, kişiler arasındaki benzer duygu yoğunlukları izlenebilmektedir.

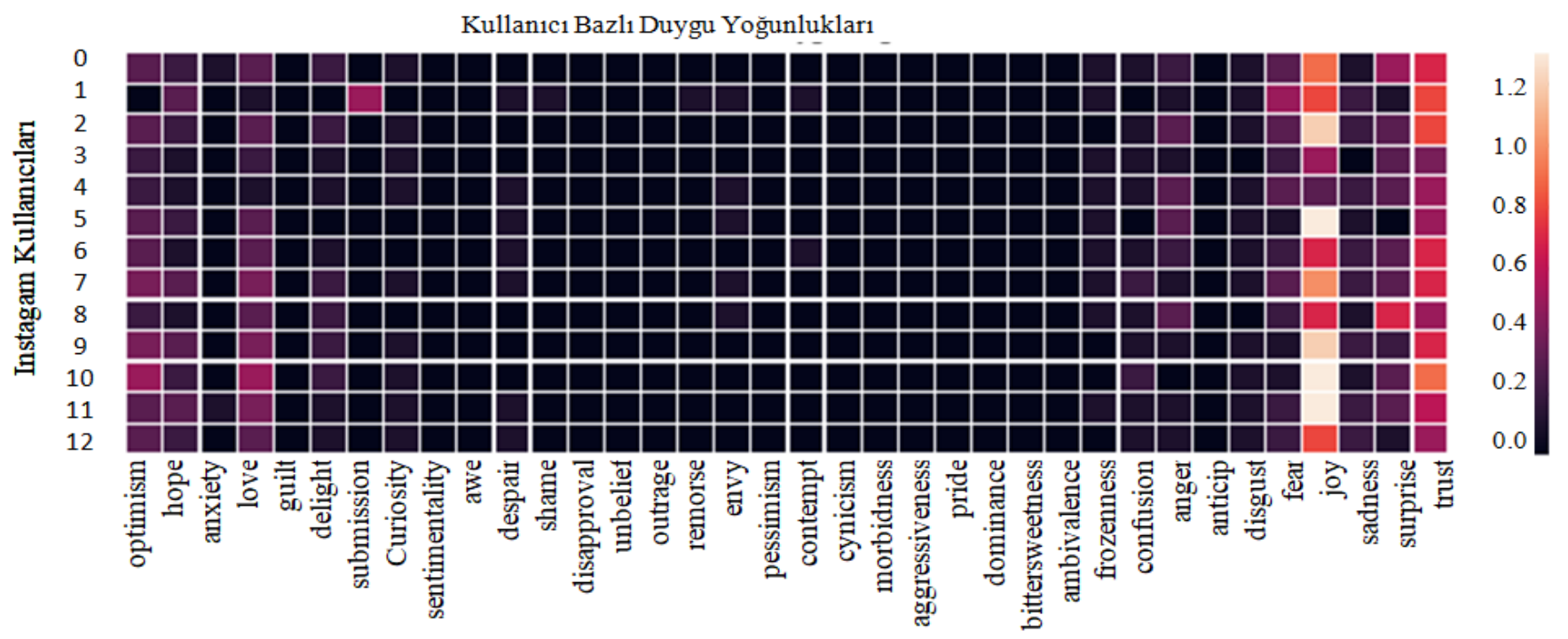

NAYALex Sözlüğü Duyguları

Şekil 3. Kullanıcı Bazlı Duygu Yoğunlukları

Örneğin 12 noluinstagram kullanıcısının veri setinde 288 farklı instagramgönderisi-paylaşımı bulunmaktadır. Veri setinde bulunan 12 no'luinstagram kullanıcısının 288 farklı paylaşımının duygu yoğunluğu bireysel olarak Şekil-4'te ele alınmıştır. İlgili kullanıcının tüm paylaşımlarını ayrı ayrı değerlendirmek yerine, ortalama duygu yoğunluğu dikkate alınarak Şekil-4'te oluşturulan 12 nolu kullanıcının en yoğun 3 duygusunun "joy (sevinç)", "trust (güven)" ve "love (sevgi)" olduğu görülmektedir.

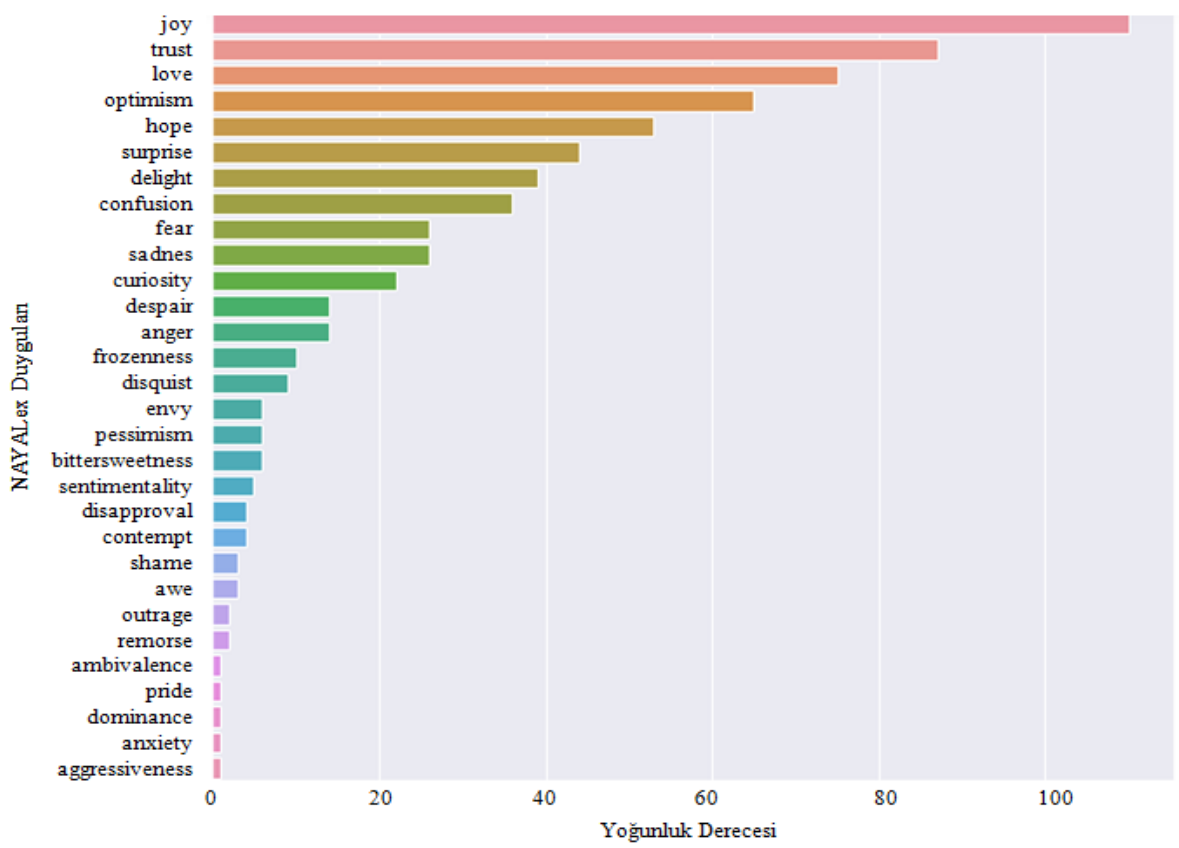

Şekil 4. 12 NoluInstagram Kullanıcısına Ait Duygu Yoğunluğu

Alșşılmış "kelime bulutu" kavramına farklı bir bakış katarak, 12 nolu kullanıcı için NAYALex Duygu Sözlüğü kullanılarak sınıflandırılmış paylaşımlarına ait örnek bir "duygu bulutu" Şekil-5'te gösterilmiştir. 


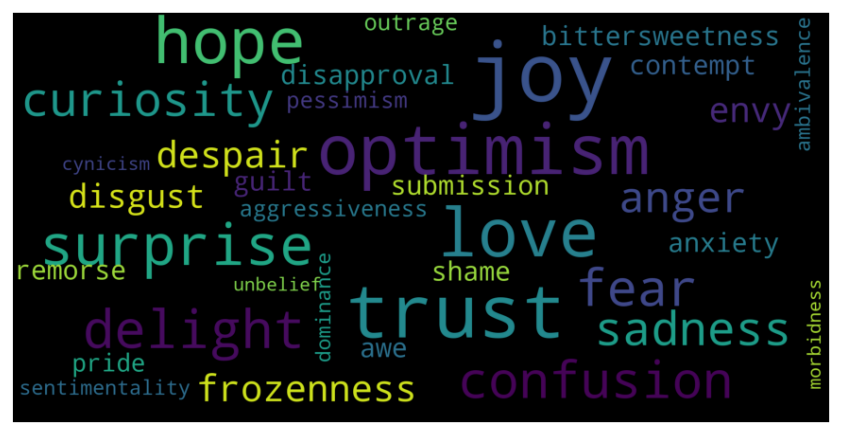

Şekil 5. 12 Nolu Kullanıcıya Ait Duygu Bulutu

Sözlük tabanlı duygu analizi için kullanılan bazı sözlükler ile NAYALex duygu sözlüğü, duygu-kapsam bakımından Tablo5 teki karşılaştırılmıștır. İfade edilen duyguların, her bir sözlük için sınıflandırılabilir olup-olmadığı dikkate alınarak ifade edilmiştir. Tablolaştırılmış duygular dikkate alındığında, kişilik üzerinde etkili olan en fazla sayıda duyguyu sinıflandırabilen sözlüğünNAYALex Duygu Sözlüğü olduğu ortaya çıkmaktadır.

Tablo 5.NAYALex ve Diğer Duygu Sözlüklerinin Duygu-Kapsam Karşılaştırması

\begin{tabular}{|c|c|c|c|c|c|}
\hline Duygu & NAYALex & $\begin{array}{c}\text { NRC } \\
\text { (Emolex) }\end{array}$ & LIWC & $\begin{array}{c}\text { EmoSentic - } \\
\text { Net }\end{array}$ & Empath \\
\hline Positive (olumlu) & $\sqrt{ }$ & $\sqrt{ }$ & $\sqrt{ }$ & $\mathrm{x}$ & $\mathrm{x}$ \\
\hline Negative (olumsuz) & $\sqrt{ }$ & $\sqrt{ }$ & $\sqrt{ }$ & $\mathrm{x}$ & $\mathrm{x}$ \\
\hline Anger (öfke) & $\sqrt{ }$ & $\sqrt{ }$ & $\sqrt{ }$ & $\sqrt{ }$ & $\sqrt{ }$ \\
\hline Sadness (üzüntü) & $\sqrt{ }$ & $\sqrt{ }$ & $\sqrt{ }$ & $\sqrt{ }$ & $\sqrt{ }$ \\
\hline Joy (sevinç) & $\sqrt{ }$ & $\sqrt{ }$ & $\mathrm{x}$ & $\sqrt{ }$ & $\sqrt{ }$ \\
\hline Fear (korku) & $\sqrt{ }$ & $\sqrt{ }$ & $\mathrm{x}$ & $\sqrt{ }$ & $\sqrt{ }$ \\
\hline Surprise (sürpriz) & $\sqrt{ }$ & $\sqrt{ }$ & $\mathrm{x}$ & $\sqrt{ }$ & $\sqrt{ }$ \\
\hline Disgust (tiksinti) & $\sqrt{ }$ & $\sqrt{ }$ & $\mathrm{x}$ & $\sqrt{ }$ & $\mathrm{x}$ \\
\hline Trust (güven) & $\sqrt{ }$ & $\sqrt{ }$ & $\mathrm{x}$ & $\mathrm{x}$ & $\mathrm{x}$ \\
\hline Anticipation (beklenti) & $\sqrt{ }$ & $\sqrt{ }$ & $\mathrm{x}$ & $\mathrm{x}$ & $\mathrm{x}$ \\
\hline Love (sevgi) & $\sqrt{ }$ & $\mathrm{x}$ & $\mathrm{x}$ & $\mathrm{x}$ & $\sqrt{ }$ \\
\hline $\begin{array}{l}\text { Optimism (iyimserlik), Hope (umut), } \\
\text { Anxiety (kaygı), Guilt (suçluluk), Delight } \\
\text { (kader), Submission (teslimiyet), Curiosity } \\
\text { (merak), Sentimentality (duygusallık), Awe } \\
\text { (huşu), Despair (umutsuzluk), Shame } \\
\text { (utanç), Bittersweetness (acı tatlılık), } \\
\text { Disapproval (onaylamama), Ambivalence } \\
\text { (kararsızlık), Unbelief (inançsızlık), Outrage } \\
\text { (öfke-nefret), Remorse (pişmanlık), } \\
\text { Envy (kıskançlık), Pessimism (karamsarlık), } \\
\text { Contempt (küçümseme), Cynicism } \\
\text { (alaycılık), Morbidness (kötülük), } \\
\text { Aggressiveness (saldırganlık), Pride (gurur), } \\
\text { Dominance (hakimiyet), Frozenness } \\
\text { (donukluk), Confusion (bilinç bulanıklı̆̆ı) }\end{array}$ & $\sqrt{ }$ & $\mathrm{x}$ & $\mathrm{x}$ & $\mathrm{x}$ & $\mathrm{x}$ \\
\hline
\end{tabular}

\section{Sonuç ve Gelecekteki Çalışmalar}

Sözlük tabanlı duygu analizi yapan çalışmalarda sınırlı sayıda duygu çıkarılması, kişiliğin belirlenmesinde yetersiz kalabilmektedir. Çalışmamızda, bu problemi iyileştirmek adına daha fazla sayıda duygu çıkarımına izin veren NAYALex Duygu
Sözlüğünü önerdik. Metinsel ifadelerin daha geniş spektrumda duygularla etiketlenmesi kişiliğin belirlenmesinde daha doğru neticeye ulaşılmasını sağlar. Bu çalışmada, Instagram veri seti üzerinde bazı deneyler yaparak, NRC duygu Sözlüğü 
(Mohammad, 2016) ve Plutchik'in Temel Duyguların Psikoevrimsel Teorisine (Drews\&Krohn, 2007) dayanarak oluşturduğumuz NAYALex'in uygulanabilirliği gösterilmiştir.

Sözlük tabanlı yaklaşımlarda, manuel olarak oluşturulan sözlüklerin yüksek maliyetinden kaçınmak için sözlüklerin otomatik bir şekilde oluşturulması önem kazanmıştır ( $\mathrm{Li}$ vd., 2012; Hatzivassiloglou\& McKeown,1997). NAYALex duygu sözlüğü, NRC duygu Sözlüğü (Mohammad, 2016) ve Plutchik'in Temel Duyguların Psikoevrimsel Teorisi (Drews\&Krohn, 2007) kullanılarak otomatik olarak oluşturulmuş olup, yüksek maliyet ortadan kaldırılmıştır.

Tablo 6. Nayalex ve Diğer Duygu Sözlüklerinin Sınıflandırma Oranlarının Karşılaştırılması(Tiffany’nin Belirttiği 154 Farklı Duyguya Göre)

\begin{tabular}{|c|c|c|c|c|c|}
\hline$\#$ & NAYALex & NRC(Emolex) & Empath & $\begin{array}{c}\text { EmoSentic- } \\
\text { Net }\end{array}$ & LIWC \\
\hline $\begin{array}{c}\text { Doğrudan } \\
\text { Sinıflandırabildiği Duygu } \\
\text { Sayıs1 }\end{array}$ & 38 & 8 & 6 & 6 & 4 \\
\hline $\begin{array}{c}\text { Tiffany'nin 154 } \\
\text { Duygusunu Temsil Etme } \\
\text { Oranı }\end{array}$ & $24,70 \%$ & $6,50 \%$ & $3,90 \%$ & $3,90 \%$ & $2,60 \%$ \\
\hline
\end{tabular}

Dünya çapında 154 farklı duygu olduğunu ifade eden Tiffany (Medhat vd., 2014)'nin belirttiği duygular arasından en fazla sayıda duygu sınıflandırması yapabilen sözlükönerdiğimiz NAYALex sözlüğüdür. Tiffany'nin belirttiği duygular dikkate alındığında; NAYALex bu duyguların \%24,7'sini, NRC \%6,5'ini, LIWC \%2,6'sını, EmoSenticNet \%3,9'unu ve Empath $\% 3,9$ 'unu oransal olarak temsil ettiği ve doğrudan sınıflandırabildiği Tablo-6 da gösterilmiştir. Bildiğimiz kadarıyla bu çalışma, sözlük tabanlı duygu sınıflandırması yapan çalışmalar arasında, doğrudan 38 farklı duyguyu ortaya çıkarabilen ve \%24,7 lik temsil oranıyla ilk en kapsamlı sözlük olmuştur.

Gelecekteki çalışmamızda NAYALex Duygu Sözlüğünü genişletmeye odaklanacağız. Bunun için WordNet vb. anlamsal sözlükler ve kelimelerin dilsel ilişkilerini kullanarak duygu sözlüğünü genişletmeye ve makine öğrenmesi tabanlı sınıflandırma algoritmalarını kullanarak test etmeye çalışacağız.

\section{Teşekkür}

Çalışmalarımda bana destek olan ve firsat veren eşim Yıldız'a, kızlarım Beyza Nur ve Meva Sultan’a teşekkür ederim.

\section{Kaynakça}

Alarcao, S. M., \&Fonseca, M. J. (2017). Emotionsrecognitionusing EEG signals: A survey. IEEE Transactions on Affective Computing, 10(3), 374-393.

Alarid, M. (2016). Recruitmentandradicalization: The role of socialmediaandnewtechnology.

Counteringillicitpower in warandtransition, 313-330.

Baccianella, S., Esuli, A., \&Sebastiani, F. (2010). Sentiwordnet 3.0:

enhancedlexicalresourceforsentimentanalysisandopinionmin ing. Lrec, 10, 2200-2204.

Bae, H. S., Lee, H. J., \& Lee, S. G. (2016, June). Voice recognitionbased on adaptive MFCC anddeeplearning. In 2016 IEEE 11th Conference on IndustrialElectronicsand Applications (ICIEA) (pp. 1542-1546). IEEE.

Breck, E., Choi, Y., \&Cardie, C. (2007, January). Identifyingexpressions of opinion in context. In IJCAI (Vol. 7, pp. 2683-2688).
Cambria, E., Schuller, B., Xia, Y., \&Havasi, C. (2013). New avenues in opinionminingandsentimentanalysis. IEEE Intelligentsystems, 28(2), 15-21.

Colombetti, $\mathrm{G}$.

(2009). Fromaffectprogramstodynamicaldiscreteemotions. PhilosophicalPsychology, 22(4), 407-425.

Deng, L., \&Wiebe, J. (2015). Mpqa 3.0: An entity/eventlevelsentimentcorpus. InProceedings of the 2015 conference of the North Americanchapter of theassociationforcomputationallinguistics: humanlanguagetechnologies (pp. 1323-1328).

Devika, M. D., Sunitha, C., \&Ganesh, A. (2016). Sentimentanalysis: a comparativestudy on differentapproaches. ProcediaComputerScience, 87, 44-49.

Drews, M. (2007). Robert Plutchik'sPsychoevolutıonaryTheory Of BasıEmotıons.(Erişim tarihi: 12.07.2021, http://www.adliterate.com/archives/Plutchik.emotion.theorie .POSTER.pdf)

Drews, M., \&Krohn, M. (2007). Robert Plutchik'sPsychoevolutionarytheory of basicemotions. University of AppliedSciencesPostdam, Germany. Retrievedfrom http://www. markusdrews. de/Plutchiks. Emotionstheorie. PLAKAT. pdf.

Er, M. B. (2020). A NovelApproachforClassification of Speech EmotionsBased on DeepandAcousticFeatures. IEEE Access, 8, 221640-221653.

ER, M. B., \& Harun, Ç. İ. Ğ. (2020). Türk Müziği Uyaranları Kullanılarak İnsan Duygularının Makine Öğrenmesi Yöntemi İle Tanınması. Gazi Üniversitesi Fen Bilimleri Dergisi Part C: Tasarım ve Teknoloji, 8(2), 458-474.

Fast, E., Chen, B., \&Bernstein, M. S. (2016, May). Empath: Understandingtopicsignals in large-scaletext. InProceedings of the $2016 \mathrm{CHI}$ conference on humanfactors in computingsystems (pp. 4647-4657).

Hatzivassiloglou, V., \&McKeown, K. (1997, July). Predictingthesemanticorientation of adjectives. In 35th annualmeeting theassociationforcomputationallinguisticsand 8th conference of theeuropeanchapter of theassociationforcomputationallinguistics (pp. 174-181).

Hidalgo, C. R., Tan, E. S. H., \&Verlegh, P. W. (2015). Thesocialsharing of emotion (SSE) in online 
socialnetworks: A casestudy in Live Journal. Computers in Human Behavior, 52, 364-372.

Hussein, D. M. E. D. M. (2018). A survey on sentimentanalysischallenges. Journal of KingSaudUniversity-EngineeringSciences, 30(4), 330-338.

Kamps, J., Marx, M., Mokken, R. J., \& De Rijke, M. (2004, May). Using WordNettomeasuresemanticorientations of adjectives. In LREC (Vol. 4, pp. 1115-1118).

Koto, F., \&Adriani, M. (2015, December). HBE: Hashtagbasedemotionlexiconsfortwittersentimentanalysis. InProceedings of the 7th Forum for Information Retrieval Evaluation (pp. 31-34).

Koumpouri, A., Mporas, I., \&Megalooikonomou, V. (2015, September). Evaluation of FourApproachesfor" Sentiment Analysis on Movie Reviews" TheKaggleCompetition. InProceedings of the 16th International Conference on Engineering Applications of Neural Networks (INNS) (pp. $1-5)$.

Kušen, E., Cascavilla, G., Figl, K., Conti, M., \&Strembeck, M. (2017, August). Identifyingemotions in socialmedia: comparison of word-emotionlexicons. In 2017 5th International Conference on Future Internet of ThingsandCloudWorkshops (FiCloudW) (pp. 132-137). IEEE.

Lang, P. J. (1995). Theemotionprobe: Studies of motivationandattention. Americanpsychologist, 50(5), 372.

Li, F., Pan, S. J., Jin, O., Yang, Q., \&Zhu, X. (2012, July). Crossdomain co-extraction of sentimentandtopiclexicons. InProceedings of the 50th Annual Meeting of theAssociationforComputationalLinguistics (Volume 1: LongPapers) (pp. 410-419).

Lin, C. K., Lee, Y. Y., Yu, C. H., \&Chen, H. H. (2014, November). Exploringensemble of models in taxonomybasedcross-domain sentimentclassification. InProceedings of the 23rd ACM International Conference on Conference on Information and Knowledge Management (pp. 12791288).

Liu, B. (2012). Sentimentanalysisandopinionmining. Synthesislectures on humanlanguagetechnologies, 5(1), 1167.

Malik, K. R., Ahmad, M., Khalid, S., Ahmad, H., Al-Turjman, F., \&Jabbar, S. (2020). Image andcommandhybrid model forvehiclecontrolusing Internet of Vehicles. Transactions on EmergingTelecommunications Technologies, 31(5), e3774.

Medhat, W., Hassan, A., \&Korashy, H. (2014). Sentimentanalysisalgorithmsandapplications: A survey. AinShamsengineeringjournal, 5(4), 1093-1113.

Mittal, S., Agarwal, S., \&Nigam, M. J. (2018, November). Real time multiplefacerecognition: A deeplearningapproach. InProceedings of the 2018 International Conference on DigitalMedicineand Image Processing (pp. 70-76).

Mohammad, S. (2011). SentimentandEmotionLexicons. ( Erişim tarihi: 12.07.2021, http://saifmohammad.com/WebPages/lexicons.html).

Mohammad, S. (2016) NRC Word-EmotionAssociationLexicon. (Erişim tarihi: 12.07.2021, http://saifmohammad.com/WebPages/NRC-EmotionLexicon.html).

Mohammad, S. M., Zhu, X., Kiritchenko, S., \& Martin, J. (2015). Sentiment, emotion, purpose, andstyle in electoraltweets. Information Processing\& Management, 51(4), 480-499.
Mohammad, S., \&Kiritchenko, S. (2013, June). Using nuances of emotiontoidentifypersonality. InSeventh International AAAI Conference on WeblogsandSocial Media.

Pang, B., \& Lee, L. (2009). Opinionminingandsentimentanalysis. Comput. Linguist, 35(2), 311-312.

Pang, B., Lee, L., \&Vaithyanathan, S. (2002). Thumbsup? Sentimentclassificationusingmachinelearningtechniques. arXivpreprintcs/0205070.

Pennebaker, J. W., Francis, M. E., \&Booth, R. J. (2001). Linguisticinquiryandwordcount: LIWC 2001. Mahway: Lawrence ErlbaumAssociates, 71(2001), 2001.

Picard, R. W. (2000). Affectivecomputing. MIT press.

Plutchik, R. (1980). A general psychoevolutionarytheory of emotion. InTheories of emotion (pp. 3-33). Academicpress.

Plutchik, R. (2001). Thenature of emotions: Human emotionshavedeepevolutionaryroots, factthatmayexplaintheircomplexityandprovidetoolsforclinica lpractice. Americanscientist, 89(4), 344-350.

Poria, S., Gelbukh, A., Cambria, E., Yang, P., Hussain, A., \&Durrani, $\quad$ T. (2012, $\quad$ October). MergingSenticNetandWordNet-

Affectemotionlistsforsentimentanalysis. In 2012 IEEE 11th internationalconference on signalprocessing (Vol. 2, pp. 1251-1255). IEEE.

Rao, D., \&Ravichandran, D. (2009, March). Semisupervisedpolaritylexiconinduction. InProceedings of the 12th Conference of theEuropeanChapter of the ACL (EACL 2009) (pp. 675-682).

Ravi, K., \&Ravi, V. (2015). A survey on opinionminingandsentimentanalysis: tasks, approachesandapplications. Knowledge-basedsystems, 89, 14-46.

Song, K., Gao, W., Chen, L., Feng, S., Wang, D., \&Zhang, C. (2016, July). Buildemotionlexiconfromthemood of crowdviatopic-assistedjointnon-negativematrixfactorization. InProceedings of the 39th International ACM SIGIR conference on Researchand Development in Information Retrieval (pp. 773-776).

Staiano, J., \&Guerini, M. (2014). Depechemood: a lexiconforemotionanalysisfromcrowd-annotatednews. arXivpreprint arXiv:1405.1605.

Turney, P. D. (2002). Thumbsuporthumbsdown? Semanticorientationappliedtounsupervisedclassification of reviews. arXivpreprintcs/0212032.

Woolf, N. (2016). As fakenewstakesover Facebook feeds, manyaretaking satire as fact. TheGuardian. Accessed, 1, 0418.

Yessenalina, A., Yue, Y., \&Cardie, C. (2010, October). Multilevelstructuredmodelsfordocument-

levelsentimentclassification. InProceedings of the 2010 conference on empiricalmethods in naturallanguageprocessing (pp. 1046-1056). 